\title{
Optimization of Scan and Reconstruction Parameters for Renal Artery CT Angiography with Iterative Reconstruction at Low kVp Compared with Filtered Back Projection at 120 kVp Acquisition
}

\author{
Pinggui Lei, Jun Jiao, ${ }^{1}$ Xiaolin Wang, $1,{ }^{1}$ Qinghong Duan, ${ }^{1}$ Xun Zou, ${ }^{1}$ Hui Feng, ${ }^{1}$ Jujiang Mao, ${ }^{1}$ and \\ Pingxian Wang ${ }^{1}$ \\ ${ }^{1}$ Department of Radiology, The Affiliated Hospital of Guizhou Medical University, Guizhou Medical University, Guiyang of Guizhou, China \\ "Corresponding author: Xiaolin Wang, Department of Radiology, The Affiliated Hospital of Guizhou Medical University. No. 28, Guiyi St, Yunyan District, Guiyang of Guizhou \\ 550004, China. Tel: +86-18786118165, E-mail:570465670@qq.com
}

Received 2017 February 12; Revised 2018 January 15; Accepted 2018 January 29.

\begin{abstract}
Background: As cross-sectional images expanded in clinical practice, the number of renal lesions discovered incidentally has been increasing. CT protocols for renal mass evaluation may include pre-and post-contrast phase. However, a major concern with respect to those multiphase techniques is often the radiation dose. One further critical issue is how to minimize the radiation dose while image quality is optimized in routine daily clinical practice.

Objectives: To evaluate the image quality and radiation dose of renal artery CT angiography (CTA) with optimal sinogram affirmed iterative reconstruction (SAFIRE) strength level at $80 \mathrm{kVp}$ for optimizing the scan and reconstruction parameters of renal artery CTA, in comparison to $120 \mathrm{kVp}$ acquisition with filtered back projection (FBP).

Patients and Methods: Sixty consecutive patients were classified into three groups prospectively in this study:1) $80 \mathrm{kVp}$ group $(\mathrm{n}=$ 30) with FBP and 2) $80 \mathrm{kVp}$ group with SAFIRE ( $\mathrm{S} 4)$ and 3) $120 \mathrm{kV}$ group $(\mathrm{n}=30)$ with FBP. CT values and noise were measured, contrastto-noise ratio (CNR) and signal-to-noise ratio (SNR) of the renal artery CTA were calculated for quantitative assessment. Subjective image quality was evaluated by two experienced abdominal radiologists with a five-point scale in a blinded and randomized setting for qualitative assessment. Volume CT dose index $\left(\mathrm{CTDI}_{\mathrm{vol}}\right)$ and size-specific dose estimate (SSDE) were calculated and estimated for each subject presented in this study.

Results: As for quantitative assessment, CNR, SNR and CT values of renal artery in 80kVp group with SAFIRE strength levels or FBP were higher than those of $120 \mathrm{kVp}$ group with FBP (all Ps $<0.05$ ), the mean noise gradually decreased in 80kVp group when increasing the SAFIRE strength levels (S1 - S5). As for qualitative assessment, the overall image quality, vessel sharpness, segmental renal artery displayed in maximum intensity projection (MIP) or multiplanar reformation (MPR), and diagnostic confidence of the two groups were all accepted by two radiologists (inter-observer agreement ranged from 0.600 to 0.940 ) (score $\geq 3$ ). The overall image quality of $80 \mathrm{kVp}$ with SAFIRE 4 was similar to that of $120 \mathrm{kVp}$ group with $\mathrm{FBP}(\mathrm{P}=0.412)$, as well as segmental renal artery displayed in MIP and diagnostic confidence $(\mathrm{P}=0.095$ and $\mathrm{P}=0.061$, respectively). As for radiation dose reduction, 80kVp group could reduce $32.7 \% \mathrm{CTDI}_{\mathrm{vol}}$ and $31.2 \% \mathrm{SSDE}$ compared to the $120 \mathrm{kVp}$ group.

Conclusion: In patients with BMI lower than $28 \mathrm{~kg} / \mathrm{m}^{2}$, renal artery CTA at $80 \mathrm{kVp}$ with SAFIRE is feasible and can provide satisfactory images for clinical applications. This method also provides reduction in radiation dose. SAFIRE strength level 4 is recommended for reconstructing renal artery CTA.
\end{abstract}

Keywords: Radiation Dose, Image Quality, Renal Artery, CT Angiography, Iterative Reconstruction and Optimization

\section{Background}

With the expansion of abdominal cross-sectional image in clinical practice, the number of renal lesions discovered daily has incidentally increased. Multidetector computed tomography (CT) is the cornerstone of imaging investigation for characterizing and evaluating renal mass discovered serendipitously (1-3). CT protocols for renal mass evaluation may include pre-contrast phase and postcontrast consisting of corticomedullary phase, nephrographic phase and excretory phase $(4,5)$. Renal artery CT angiography (CTA), equivalent to the image of corticomedullary phase, is often performed to provide additional valuable information for surgical planning and assessment of the renal artery anatomy (5). However, a major 
concern with respect to those multiphase techniques is often the involvement of a higher radiation dose, which have indicated to be associated with increased cancer risks by other studies (6); One further critical issue is how to minimize the radiation dose for patients and provide high image quality to radiologists for the diagnosis.

Compared with the traditional filtered back projection (FBP) reconstruction method, iterative reconstruction algorithms can improve image quality by using multiple reconstruction iterations to decrease the artificial and image noise without degrading spatial resolution and compromising the overall image quality. Therefore, sinogram affirmed iterative reconstruction (SAFIRE) as a new technique applying iterative reconstruction algorithms has been implemented in abdominal CT angiography to improve image quality by reducing image noise. Initial results demonstrated that the iterative reconstruction might improve the feasibility at low tube voltage $(7,8)$. Such a technique provides the potential to reduce the radiation dose for patients suspected with renal lesion in CT examinations and facilitates respective clinical diagnosis and treatment.

\section{Objectives}

Based on the novel technique of iterative reconstruction, the purpose of this study was to investigate the image quality, radiation dose, feasibility for renal artery CTA at $80 \mathrm{kVp}$ among patients performed abdominal CT examination in comparison to a standard $120 \mathrm{kVp}$ acquisition with FBP, and optimizing the scan and reconstruction parameters for renal artery CTA.

\section{Patients and Methods}

\subsection{Patients}

This prospective study obtained institutional review board approval at the Affiliated Hospital of Guizhou Medical University (AHGMU). Sixty patients performed abdominal CT examination were enrolled into this study from April 2015 to March 2016 in AHGMU.

Inclusion criteria: 1 , Patients suspected with renal mass or abdominal pain by clinic; 2 , Patients age: 18 - 80 years; 3 , Body mass index (BMI) of each patient was lower than 28 $\mathrm{kg} / \mathrm{m}^{2}$.

Exclusion criteria: 1, Patients with renal mass after surgery; 2, Patients with the history of serious hypersensitivity reaction to contrast media; 3 , Patients with renal insufficiency (estimated glomerular filtration rate (eGFR) $<60 \mathrm{~mL} / \mathrm{min} / 1.73 \mathrm{~m}^{2}$ ); 4, Insufficient cardiac function; 5,
Inability to hold breath to meet CTA requirement; 6, Patients with manifest thyrotoxicosis; 7, Pregnant women; 8 , Patients who underwent other clinicals trial at the same time; 9, Inability to meet study requirements during experiments.

Randomization procedure: Assign $80 \mathrm{kVp}$ or $120 \mathrm{kVp} \mathrm{CT}$ protocol to patients randomly according to the order of enrolment in this prospective study, the randomization procedure was based on the random number and block randomization by using SPSS software (version 23.0).

\subsection{CT Protocols for Renal Artery CTA}

Sixty consecutive patients underwent 128-slice multidetector CT (MDCT) scanning (SOMATOM definition AS+, Siemens, Germany).

For the $80 \mathrm{kVp}$ group, the settings were as follows: tube voltage $80 \mathrm{kVp}$, tube current 400 - $500 \mathrm{mAs}$, collimation $128 \times 0.6 \mathrm{~mm}, 1 \mathrm{~mm}$ slice thickness, $500 \mathrm{~mm}$ field of view (FOV);

For the $120 \mathrm{kVp}$ group, the settings were: tube voltage $120 \mathrm{kVp}$, tube current and collimation were 200 - $250 \mathrm{mAs}$ and $128 \times 0.6 \mathrm{~mm}, 1 \mathrm{~mm}$ slice thickness, $500 \mathrm{~mm}$ FOV.

CT image datasets were reconstructed with SAFIRE at $80 \mathrm{kVp}$, and with FBP at $120 \mathrm{kVp}$. The acquisition followed craniocaudal direction, while breath holding, a series of unenhanced images of the upper abdomen were taken to evaluate the position of the renal region. The data of renal artery CTA were obtained at 25 - 30 seconds after administration of contrast medium (iopromide, $370 \mathrm{mgI} / \mathrm{mL}, 80$ $\mathrm{mL}$ ) injection at a flow rate of $3.0 \mathrm{~mL} / \mathrm{s}$ by high pressure injector at the left superficial vein in the antecubital fossa. The scan parameters included a rotation time of $0.5 \mathrm{sec}$ onds, a pitch of 0.6 and a matrix of $512 \times 512$.

\subsection{CT image Reconstruction}

For renal artery CTA, all the CT images datasets were reconstructed at the workstation (Syngo CT Workplace VE40B), different iterative reconstruction strength levels and FBP algorithm were also reconstructed based on its original datasets. CT images with $1 \mathrm{~mm}$ thickness and no intersection gap were generated for renal artery CTA. Multiplanar reformation (MPR) images and maximum intensity projection (MIP) images (including axial, sagittal, coronal image) were also generated, and these images were subsequently loaded into picture archiving and communication system (PACS) workstation (Syngo.plaza, Siemens) for evaluation by two experienced abdominal radiologists with a five-point scale. 


\subsection{Quantitative Assessment for Renal Artery CTA}

With regards to the quantitative assessment of renal artery CTA, the signal intensity measurement was performed for each patient in corticomedullary phase contrast enhanced image datasets at workstation by means of Hounsfield units (HU). The CT attenuation of abdominal aorta (AO), right renal artery (RRA), left renal artery (LRA), erector spinae major (ESM), fat of subcutaneous abdominal wall (FSAW) and air were measured by placing the regions of interest (ROI) on the axial images. ROIs were drawn as large as the vessel lumen, and avoided the calcification of lumen wall or artifacts. Standard deviation (SD) of CT attenuation of air at the level of renal artery about $1 \mathrm{~cm}$ distal to the anterior abdominal skin was defined as background noise. Depending on the data of CT value, contrast to noise ratio (CNR) and signal to noise ratio (SNR) were calculated using the following formula:

$C N R_{\text {vessels }}=\frac{C T_{\text {vessels }}-C T_{\text {muscle }}}{S D}$

$S N R_{\text {vessels }}=\frac{C T_{\text {vessels }}}{S D}$

Where $\mathrm{CT}_{\text {vessels }}$ is the $\mathrm{CT}$ value of renal artery and other vessels, $\mathrm{CT}_{\text {muscle }}$ is the $\mathrm{CT}$ value of erector spinae major, $\mathrm{SD}$ is standard deviation of CT value of air.

\subsection{Qualitative Assessment for Renal Artery CTA}

All the MPR and MIP CTA images were evaluated by two experienced abdominal radiologists with 10 and 11 years experience who were blinded to the CT scanning parameters reviewed with a five-point scale.

Image quality was scored as follows:

For the overall image:

score of $1=$ poor;

score of 2 = suboptimal;

score of 3 = diagnostic;

score of $4=$ superior;

score of $5=$ excellent.

Image quality was scored as follows for the sharpness of renal artery: tail;

score of $1=$ unacceptable anatomical structure and de-

score of 2 = poor anatomical structure and detail;

score of 3 = acceptable anatomical structure and detail;

score of $4=$ preferable sharpness of anatomical structure and detail;

score of $5=$ ideal sharpness of anatomical structure and detail.

The detail of image quality was scored as follows for the segment of renal artery displayed in MPR and MIP image: score of $1=$ main renal artery;

score of 2 = first segment of renal artery;

score of $3=$ second segment of renal artery;

score of $4=$ third segment of renal artery;

score of $5=$ four segment of renal artery.

Diagnostic confidence was also evaluated by a fivepoint scale:

score of $1=$ very poor confidence,

score of 2 = suboptimal confidence,

score of $3=$ acceptance confidence,

score of $4=$ superior confidence,

score of $5=$ excellent confidence.

The score of image quality $\geq 3$ was satisfactory with clinical application.

\subsection{Radiation Dose Assessment}

Volume CT dose index $\left(\mathrm{CTDI}_{\mathrm{vol}}\right)$ and dose length product (DLP) were recorded according to the radiation dose report in the CT scanning. Effective dose (ED) and sizespecific dose estimate (SSDE) were calculated for each patient according to the size of the body, including the size of lateral (LAT) dimension and anterior-posterior (AP) dimension at the level of the renal artery. Percentage dose reduction (DR) ratio was also computed in accordance with the following formula:

$$
\begin{aligned}
& D R_{C T D I} \%=\frac{C T D I_{120 \mathrm{kvp}}-C T D I_{80 \mathrm{kvp}}}{C T D I_{120 \mathrm{kVp}}} \times 100 \% \\
& D R_{S S D E} \%=\frac{S S D E_{120 \mathrm{kvp}}-S S D E_{80 \mathrm{kVp}}}{S S D E_{120 \mathrm{kVp}}} \times 100 \% \\
& S S D E=f_{\text {size }}^{32 X} \times C T D I_{v o l}(m G y) \\
& E D=k\left(m S v \times m G y^{-1} \times \mathrm{cm}^{-1}\right) \times D L P(m G y \times \mathrm{cm})
\end{aligned}
$$

Where $f_{\text {size }}^{32 X}$ is the size-specific body radiation dose estimate conversion factor (9), $\mathrm{CTDI}_{12 \mathrm{kVp}}$ is $\mathrm{CTDI}_{\mathrm{vol}}$ valued at $120 \mathrm{kVp}$ and $\mathrm{CTD}_{\mathrm{I} 0 \mathrm{kVp}}$ is $\mathrm{CTDI}_{\mathrm{vol}}$ valued at $80 \mathrm{kVp}$. $S S D E_{120 \mathrm{kVp}}$ is considered as the mean value of SSDE in $120 \mathrm{kVp}$ group, $\mathrm{SSDE}_{80 \mathrm{kVp}}$ is considered as the mean value in $120 \mathrm{kVp}$ group, $\mathrm{k}$ refers to the body region, age and $\mathrm{kV}$-specific dose conversion factor (10).

\subsection{Statistical Analysis}

A statistical analysis was performed by utilizing SPSS software for Windows (version 23.0, SPSS Inc., Chicago, IL). Continuous variables were expressed as mean \pm SD. The normality assumption of variables was conducted according to the Kolmogorow-Smirnov Z test. Categorized variable (gender) was analyzed by Chi-square test. Age was 
executed by the independent-sample $T$ test. As for the 80 $\mathrm{kVp}$-FBP group, $80 \mathrm{kVp}-\mathrm{S} 4$ and $120 \mathrm{kVp}-\mathrm{FBP}$, the CT attenuation of AO, RRA, LRA, FSAW, AIR, ESM, SD, CNR and SNR of renal artery were analyzed by two-tailed paired-samples $T$ test or independent-sample T test. Qualitative score was performed by Mann-Whitney U or Wilcoxon test among qualitative variables (overall image quality, vessels sharpness, segmental displayed in MIP or MPR, diagnostic confidence). As for the differences of subgroups (S1 - S5) at 80 $\mathrm{kVp}$, quantitative assessment and qualitative assessment were both determined with the nonparametric Friedman's test and performed pairwise comparisons, the significant difference level was adjusted. Radiation dose of the $80 \mathrm{kVp}$ groups and $120 \mathrm{kVp}$ group was tested by two-tailed, unpaired t-test.

Kappa analysis and percentage agreement were considered to evaluate the qualitative analysis and performed through MedCalc software (MedCalc 15.2.2, Mariakerke, Belgium). Definitions of inter-observer agreement on the basis of $k$ value were as follows:

$\mathrm{k}$ values less than 0.20 were an indication of poor strength agreement,

$\mathrm{k}$ values between 0.21 and 0.40 were an indication of fair strength of agreement,

$\mathrm{k}$ values between 0.41 and 0.80 were an indication of moderate strength of agreement,

$\mathrm{k}$ values between 0.81 and 1.00 were an indication of very good strength of agreement.

$\mathrm{P}<0.05$ was considered as statistically significant.

\section{Results}

\subsection{Patients Characteristics}

Imaging evaluation of all 60 patients was performed with multislice CT at $80 \mathrm{kVp}$ or $120 \mathrm{kVp}$ acquisition. There were no significant differences in the patients' age ( $\mathrm{P}=$ 0.205 ), as well as sex between $80 \mathrm{kVp}$ group (male 13, female 17) and $120 \mathrm{kVp}$ group (male 14 , female 16$)(\mathrm{P}=1.000)$. There were 35 patients diagnosed with renal cyst by ultrasound, six patients had a renal lesion by lumbar vertebra CT examination, seven patients had an abnormal intensity signal of the kidney by lumbar vertebra MRI examination, 12 patients were suspected of a renal mass by means of hematuria.

\subsection{Radiation Dose Assessment}

According to the CT protocol we performed in this study, the CTDI ${ }_{\mathrm{vol}}$ for renal artery CTA at $80 \mathrm{kVp}$ was much lower compared with those of $120 \mathrm{kVp}$ acquisition ((10.1 \pm 1.2) mGy vs. (15.0 \pm 2.6$) \mathrm{mGy}, \mathrm{P}<0.001)$. The dose reduction of CTDI ${ }_{\mathrm{vol}}$ was $32.7 \%$, the dose reduction of SSDE was 31.2\% (P
$<0.001)$. The dose reduction of ED and DLP was $40.0 \%$ and $39.6 \%$, respectively (all Ps $<0.001$ ).

\subsection{Quantitative Analysis for Image Quality}

As shown in Table 1 and Table 2, image quality was analyzed through detailed data for quantitative analysis. A significant statistical difference can be seen in terms of the mean CT value of AO, RRA, LRA, ESM and the SD of air for 80 $\mathrm{kVp}$ group with iterative reconstruction strength level 4 or with FBP versus $120 \mathrm{kVp}$ group with FBP (All Ps < 0.05) (Table 1). The CT values of AO, RRA, LRA, ESM within the $80 \mathrm{kVp}$ group were higher than the CT values of those in the 120 $\mathrm{kVp}$ group. The mean noise (SD of air) gradually decreased in the $80 \mathrm{kVp}$ subgroups when increasing iterative reconstruction strength levels (S1 - S5) (Table 2). Compared with the $120 \mathrm{kVp}$ group, the noise of $80 \mathrm{kVp}$ with iterative reconstruction level 4 was lower than that of $120 \mathrm{kVp}$ with FBP (P $<0.001)$. However, the noise of $80 \mathrm{kVp}$ with FBP was higher than that of $120 \mathrm{kVp}$ with $\mathrm{FBP}(\mathrm{P}<0.001)$ (Table 1$)$. For CNR and SNR of RRA, and LRA, a significant statistical difference in terms of CNR and SNR between the $80 \mathrm{kVp}$ group conducted with iterative reconstruction strength level 4 or FBP and $120 \mathrm{kVp}$ group with FBP, CNR and SNR values of RRA, and LRA within the $80 \mathrm{kVp}$ groups were higher than CNR and SNR values of those in the $120 \mathrm{kVp}$ group. The mean CNR and SNR values gradually increased with increasing iterative reconstruction strength levels (Table 2) (Figure 1).

\subsection{Qualitative Analysis for Image Quality}

For the qualitative analysis regarding image quality at $80 \mathrm{kVp}$ and $120 \mathrm{kVp}$, the subjective image was evaluated by two experienced abdominal radiologists with a five-point scale. The overall image quality, vessel sharpness, segmental arteries displayed in MIP and MPR, and diagnostic confidence are summarized in Table 3. Inter-observer agreement was established between the two experienced radiologists $(\mathrm{k}=0.600-\mathrm{k}=0.940)$ (Table 4$)$. The image quality of three groups was proved sufficient to support clinical diagnosis. The noise decreased when the iterative reconstruction strength levels increased. However, the "paintbrushed" artifact increased (Figure 2).

\section{Discussion}

The results observed in this study indicated that the renal artery CTA at $80 \mathrm{kVp}$ is feasible for patients with suspected renal mass whose BMI is less than $28 \mathrm{~kg} / \mathrm{m}^{2}$. Such configuration is believed to be able to provide satisfactory images for clinical application with $32.7 \% \mathrm{CTDI}_{\mathrm{vol}}$ and $31.2 \%$ 


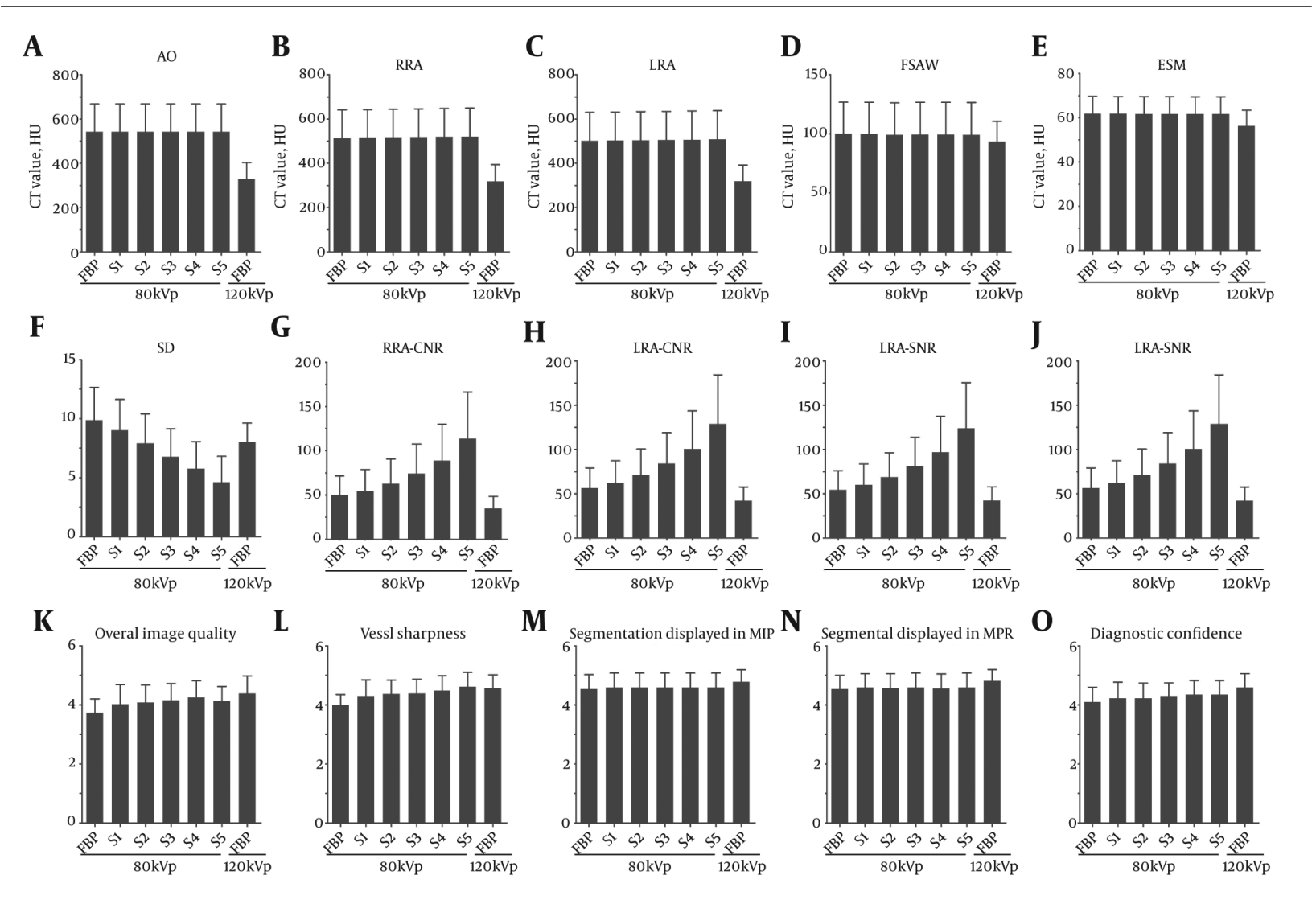

Figure 1. Comparison of quantitative and qualitative assessment among $80 \mathrm{kVp}$-filtered back projection (FBP), $80 \mathrm{kVp}$-sinogram affirmed iterative reconstruction (SAFIRE) and $120 \mathrm{kVp}$-FBP. The CT values of abdominal aorta (AO), right renal artery (RRA), left renal artery (LRA), erector spinae major (ESM) were higher at $80 \mathrm{kVp}$ than CT values of those at $120 \mathrm{kVp}(\mathrm{A}-\mathrm{E})$. The image noise decreased in the $80 \mathrm{kVp}$ subgroups (S1 - S5) with increasing SAFIRE strength levels (F). However, the contrast to noise ratio (CNR) and signal to noise ratio (SNR) of renal artery increased in the $80 \mathrm{kVp}$ subgroups with increasing SAFIRE strength levels (G-J). The overall image quality, vessel sharpness, segmental renal artery displayed in maximum intensity projection (MIP) and multiplanar reformation images (MPR) (K-N) were satisfactory with clinical practice (O).

SSDE radiation dose reduction. The optimal iterative reconstruction at level 4 could be recommended as future CT protocols for $80 \mathrm{kVp}$ renal artery CTA.

The radiology community currently implements radiation dose of CT management procedures corresponding to the principle of ALARA (as low as reasonably achievable) (11-13). Therefore, the mechanisms for reducing the radiation dose at MDCT may include X-ray beam collimation, $\mathrm{X}$-ray beam filtration, tube current modulation and adaptation for patient body habitus (automatic exposure control) and peak kilovoltage optimization, to improve the efficiency of the detection system and the noise reduction algorithms $(14,15)$. In our study, in order to obtain the relatively higher overall image quality with noise reduction algorithm technique of iterative reconstruction for diagnosis, we increased the tube current that was up to $400-500$ mAs.

In this study, the noise reduction algorithms (iterative reconstruction) were adopted to provide high image qual- ity of renal artery CT angiography at $80 \mathrm{kVp}$, while the radiation dose was reduced among patients with suspected renal mass. $\mathrm{CTDI}_{\mathrm{vol}}$ dose for the $80 \mathrm{kVp}$ group had a $32.7 \%$ reduction, in comparison to the $120 \mathrm{kVp}$ group. SSDE of each patient was calculated according to the size of the body for each patient, and the transverse and anteroposterior diameters of renal artery were both taken into consideration. SSDE value of (7.20 \pm 0.27$) \mathrm{mGy}$ for the $80 \mathrm{kVp}$ group had a $31.2 \%$ SSDE reduction in comparison to the $120 \mathrm{kVp}$ group. Additionally, in comparison to the $120 \mathrm{kVp}$ group, this study observed a reduction of approximately 39.6\% DLP and $40.0 \%$ ED in the $80 \mathrm{kVp}$ group through iterative reconstruction. Thus, it is assumed that the CT images at $80 \mathrm{kVp}$ with iterative reconstruction could provide satisfactory image quality for renal artery CTA (Figure 2).

Recent research and literature on radiation dose and image quality with iterative reconstruction have suggested that SAFIRE can improve the image quality, and has the potential to reduce the radiation dose (16-20). In our 

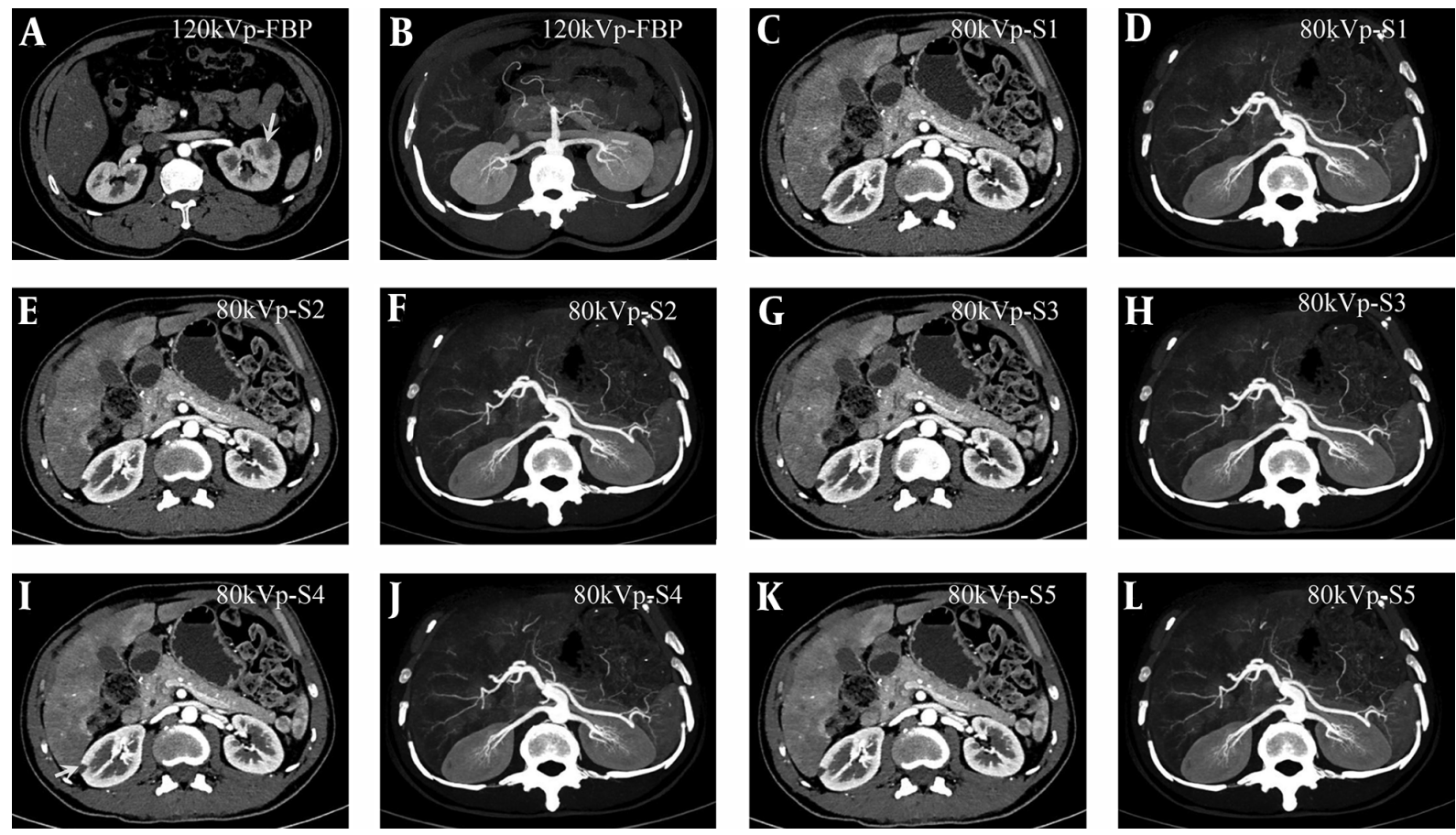

Figure 2. Comparison of image quality between $120 \mathrm{kVp}(\mathrm{A}$ and $\mathrm{B})$ and $80 \mathrm{kVp}$ different strength levels (C - $\mathrm{L}$ ) in terms of the subjective image quality of renal artery CT angiography (CTA). For the overall image quality, the renal artery CTA reconstructed by filtered back projection (FBP) at $120 \mathrm{kVp}$ and by sonogram affirmed iterative reconstruction (SAFIRE) at $80 \mathrm{kVp}$ could be accepted for clinical application (score $\geq 3$ ). However, the image quality at $80 \mathrm{kVp}$ presented more high-contrast than that at $120 \mathrm{kVp}$. Comparison was done between the two reconstruction methods for the main and segmental renal artery displayed in CT maximum intensity projection (MIP) or multiplanar reformation (MPR) images, most of the third segmental renal artery could be displayed. As for image noise, the noise decreased when strength levels were increased. However, "paint-brushed" artifact increased. Note: the renal lesion could be detected in the CT image with SAFIRE (I) or $120 \mathrm{kVp}$-FBP (A) (white arrow). 


\begin{tabular}{|c|c|c|c|c|c|c|}
\hline \multirow[t]{2}{*}{ Subject } & \multicolumn{2}{|c|}{$80 \mathrm{kVp}$} & \multirow{2}{*}{$\begin{array}{c}120 \mathrm{kVp} \\
\text { FBP }\end{array}$} & \multirow{2}{*}{$\begin{array}{c}\text { P value } \\
80 \text { kVp-FBP vs. } 80 \text { kVp-S4 }\end{array}$} & \multirow{2}{*}{$\begin{array}{c}\text { P value } \\
\text { 80 kVp-FBP vs. } 120 \\
\text { kVp-FBP }\end{array}$} & \multirow{2}{*}{$\begin{array}{c}\text { P value } \\
\text { 80 kVp-S4 vs. } 120 \\
\text { kVp-FBP }\end{array}$} \\
\hline & FBP & S4 & & & & \\
\hline AO & $542.4 \pm 125.3$ & $542.6 \pm 125.1$ & $328.8 \pm 75.0$ & $0.230^{\mathrm{b}}$ & $<0.001^{\mathrm{c}}$ & $<0.001^{\mathrm{C}}$ \\
\hline RRA & $514.1 \pm 126.7$ & $519.4 \pm 127.9$ & $317.8 \pm 76.6$ & $<0.001^{\mathrm{b}}$ & $<0.001^{\mathrm{C}}$ & $<0.001^{\mathrm{c}}$ \\
\hline LRA & $501.3 \pm 128.7$ & $506 \pm 130.1$ & $319.2 \pm 72.9$ & $<0.001^{\mathrm{b}}$ & $<0.001^{\mathrm{c}}$ & $<0.001^{\mathrm{c}}$ \\
\hline FSAW & $-99.8 \pm 26.8$ & $-99.2 \pm 27.3$ & $-93.1 \pm 17.3$ & $0.055^{\mathrm{b}}$ & $0.028^{c}$ & $0.091^{c}$ \\
\hline AIR & $-1000.8 \pm 2.8$ & $-999.6 \pm 2.6$ & $-1001.1 \pm 2.1$ & $<0.001^{\mathrm{b}}$ & $0.684^{c}$ & $0.023^{c}$ \\
\hline ESM & $61.7 \pm 7.8$ & $61.7 \pm 7.8$ & $56.2 \pm 7.2$ & $0.746^{\mathrm{b}}$ & $0.007^{c}$ & $0.007^{c}$ \\
\hline SD & $9.9 \pm 2.8$ & $5.8 \pm 2.3$ & $8.0 \pm 1.6$ & $<0.001^{\mathrm{b}}$ & $<0.001^{\mathrm{C}}$ & $<0.001^{\mathrm{c}}$ \\
\hline RRA-CNR & $49.67 \pm 21.9$ & $88.8 \pm 41$ & $34.8 \pm 13.6$ & $<0.001^{\mathrm{b}}$ & $0.008^{c}$ & $<0.001^{\mathrm{c}}$ \\
\hline RRA_SNR & $56.3 \pm 22.9$ & $100.6 \pm 43$ & $42.3 \pm 15.4$ & $<0.001^{\mathrm{b}}$ & $0.016^{c}$ & $<0.001^{\mathrm{c}}$ \\
\hline LRA-CNR & $47.8 \pm 20.7$ & $85.2 \pm 38.7$ & $35.1 \pm 13.6$ & $<0.001^{\mathrm{b}}$ & $0.019^{c}$ & $<0.001^{\mathrm{c}}$ \\
\hline LRA-SNR & $54.4 \pm 21.5$ & $97 \pm 40.5$ & $42.5 \pm 15.4$ & $<0.001^{\mathrm{b}}$ & $0.035^{\mathrm{c}}$ & $<0.001^{\mathrm{c}}$ \\
\hline Overall image quality & $3.7 \pm 0.5$ & $4.3 \pm 0.6$ & $4.4 \pm 0.6$ & $0.001^{\mathrm{d}}$ & $<0.001^{\mathrm{e}}$ & $0.412^{\mathrm{e}}$ \\
\hline Vessel sharpness & $4.0 \pm 0.3$ & $4.5 \pm 0.5$ & $4.6 \pm 0.4$ & $<0.001^{\mathrm{d}}$ & $<0.001^{\mathrm{e}}$ & $0.515^{\mathrm{e}}$ \\
\hline $\begin{array}{l}\text { Segmental dislayed in } \\
\text { MIP }\end{array}$ & $4.5 \pm 0.5$ & $4.6 \pm 0.5$ & $4.8 \pm 0.4$ & $0.083^{d}$ & $0.035^{\mathrm{e}}$ & $0.095^{\mathrm{e}}$ \\
\hline $\begin{array}{l}\text { Segmental dislayed in } \\
\text { MPR }\end{array}$ & $4.5 \pm 0.5$ & $4.6 \pm 0.5$ & $4.8 \pm 0.4$ & $0.564^{d}$ & $0.011^{\mathrm{e}}$ & $0.026^{\mathrm{e}}$ \\
\hline Diagnostic confidence & $4.1 \pm 0.5$ & $4.4 \pm 0.5$ & $4.6 \pm 0.5$ & $0.010^{d}$ & $<0.001^{\mathrm{e}}$ & $0.061^{e}$ \\
\hline
\end{tabular}

Abbreviation: AO, Abdominal aorta; CNR, Contrast-to-noise ratio; ESM, Erector spinae major; FBP, Filtered back projection; FSAW, Fat of subcutaneous abdominal wall; LRA, Left renal artery; MIP, Maximum intensity projection; MPR, Multiplanar reformation images; RRA, Right renal artery; S1-S5, SAFIRE strength level 1-5; SD, Standard deviation; SNR, Signal-to-noise ratio.

${ }^{\mathrm{a}}$ Values are expressed as mean $\pm \mathrm{SD}$.

${ }^{\mathrm{b}}$ Determined with the paired-samples $\mathrm{T}$ test.

${ }^{\mathrm{c}}$ Determined with the independent-sample $\mathrm{T}$ test.

${ }^{\mathrm{d}}$ Determined with Wilcoxon test.

${ }^{\mathrm{e}}$ Determined with Mann-Whitney U test.

study, there was significant difference found in terms of CNR and SNR of renal artery CTA between standard FBP reconstruction at $120 \mathrm{kVp}$ and SAFIRE reconstruction at 80 $\mathrm{kVp}$. With regards to the $\mathrm{CT}$ attenuation, which mainly relates to the tube voltage, the $\mathrm{CT}$ value is considerably larger in the lumen of the renal artery at $80 \mathrm{kVp}$ than those at 120 $\mathrm{kVp}$. Regarding image noise in this study, SAFIRE, as a novel reconstruction technique, is a raw-data-based iterative reconstruction algorithm that compares reconstructed and measured CT data in the raw data domain and iteratively corrects the images $(21,22)$. However, image noise of renal artery CTA showed a considerably higher amount at $80 \mathrm{kVp}$ than performed at $120 \mathrm{kVp}$, and it is believed that SAFIRE reconstruction can reduce the image noise at $80 \mathrm{kVp}$, and provide higher image quality of renal artery for patients with suspected renal mass at $80 \mathrm{kVp}$ for clinical practice. In addition, previous studies used SAFIRE strength level of 3 to investigate low radiation dose and image quality in the abdomen $(7,19)$. To our knowledge, this study has been the first attempt to focus on adopting different SAFIRE strength levels to achieve the optimal iterative reconstruction level based on raw data for evaluating the renal artery 


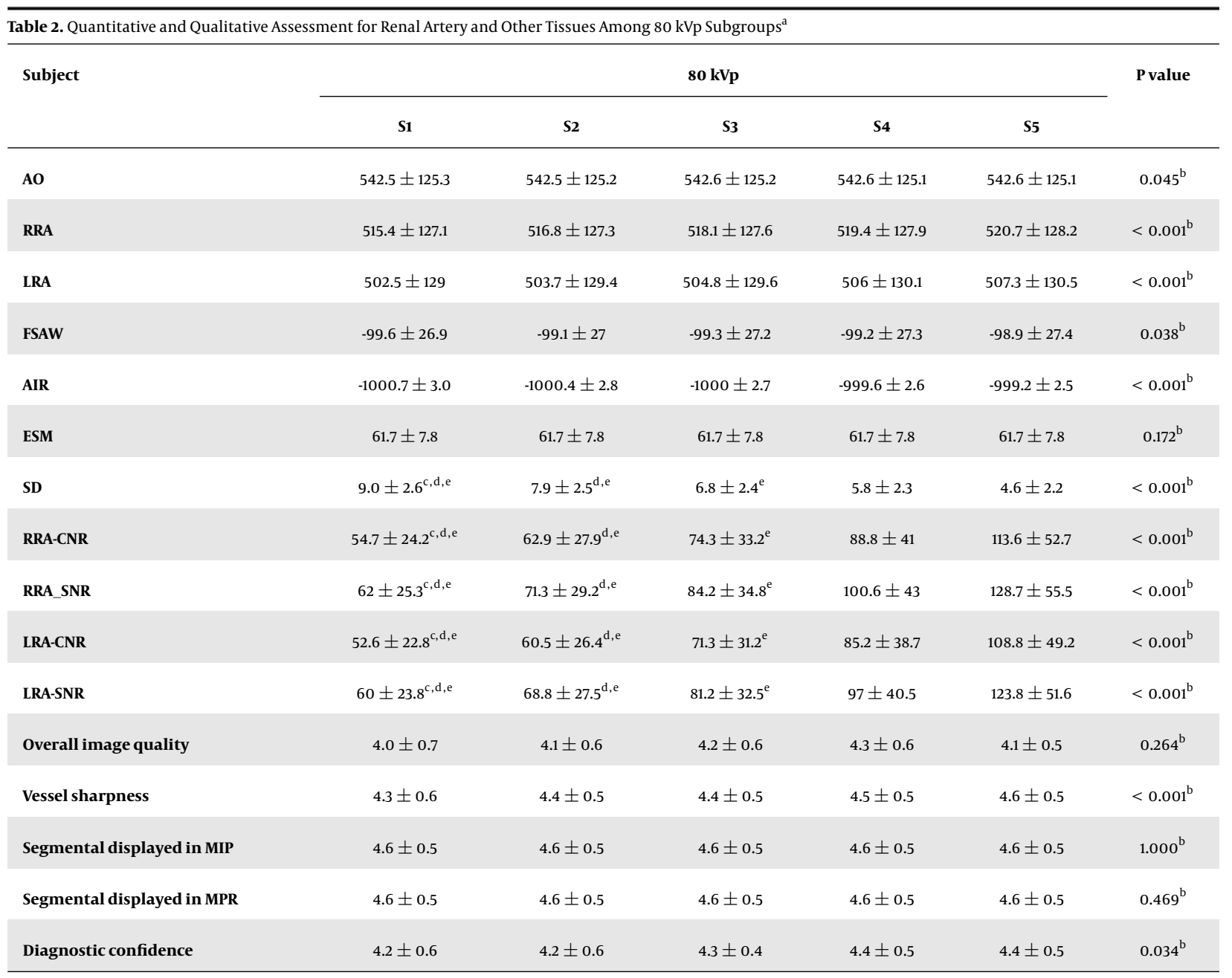

Abbreviation: AO, Abdominal aorta; CNR, Contrast-to-noise ratio; ESM, Erector spinae major; FBP, Filtered back projection; FSAW, Fat of subcutaneous abdominal wall; LRA, Left renal artery; MIP, Maximum intensity projection; MPR, Multiplanar reformation images; RRA, Right renal artery; S1-S5, SAFIRE strength level 1-5; SD, Standard deviation; SNR, Signal-to-noise ratio.

${ }^{a}$ Values are expressed as mean $\pm S D$.

${ }^{\mathrm{b}}$ Determined with the nonparametric Friedman's test and performed pairwise comparisons among 80 kVp subgroups (S1 - S5), the significant difference level was adjusted.

${ }^{\mathrm{C}}$ In comparison to $\mathrm{S} 3, \mathrm{P}<0.001$

${ }^{\mathrm{d}}$ In comparison to $\mathrm{S} 4, \mathrm{P}<0.001$

${ }^{\mathrm{e}}$ In comparison to $\mathrm{S} 5, \mathrm{P}<0.001$

CTA. Our study results showed that CNR and SNR of the renal artery increased and the image noise decreased in the $80 \mathrm{kVp}$ subgroups (S1 - S5). With increasing SAFIRE strength levels, the impression of CT images could appear "paintbrushed" or smooth overly $(15,20,23)$. However, there was a little difference about the CT attenuation of abdominal aorta, right renal artery, left renal artery, erector spinae major, fat of subcutaneous abdominal wall and air. There was no significant statistical difference in the segmental renal artery displayed in MIP or MPR images. Therefore, this study concludes that the optimal SAFIRE strength level 4 can be recommended for renal artery CTA according to our results and experience and in daily clinical practice.

There are limitations for this study. First, the sample size in this study was relatively small and we did not include patients with higher BMIs. The results would have been more useful if we included patients whose BMIs were higher than $28 \mathrm{~kg} / \mathrm{m}^{2}$ and formulated personalized CT protocols for renal artery CTA in the patients with renal mass based on the novel technique of iterative reconstruction. Second, the comparison between image quality of $80 \mathrm{kVp}$ and that of $120 \mathrm{kVp}$ was not conducted through the same individual subject.

In conclusion, using iterative reconstruction at a low 


\begin{tabular}{|c|c|c|c|c|c|c|c|c|c|c|}
\hline \multirow{2}{*}{$\begin{array}{l}\text { Reconstruction } \\
\text { methods }\end{array}$} & \multicolumn{5}{|c|}{ Reader 1} & \multicolumn{5}{|c|}{ Reader 2} \\
\hline & $\begin{array}{l}\text { Overall } \\
\text { image } \\
\text { quality }\end{array}$ & $\begin{array}{c}\text { Vessel } \\
\text { sharp- } \\
\text { ness }\end{array}$ & $\begin{array}{c}\text { Segmental } \\
\text { displayed } \\
\text { in MIP }\end{array}$ & $\begin{array}{l}\text { Segmental } \\
\text { displayed } \\
\text { in MPR }\end{array}$ & $\begin{array}{l}\text { Diagnostic } \\
\text { confi- } \\
\text { dence }\end{array}$ & $\begin{array}{l}\text { Oerall } \\
\text { image } \\
\text { quality }\end{array}$ & $\begin{array}{c}\text { Vessel } \\
\text { sharp- } \\
\text { ness }\end{array}$ & $\begin{array}{l}\text { Segmental } \\
\text { displayed } \\
\text { in MIP }\end{array}$ & $\begin{array}{c}\text { Segmental } \\
\text { displayed } \\
\text { in MPR }\end{array}$ & $\begin{array}{l}\text { Diagnostic } \\
\text { confi- } \\
\text { dence }\end{array}$ \\
\hline \multirow{3}{*}{$120 \mathrm{kVp}$-FBP } & $3(1 / 30)$ & & & & & $3(2 / 30)$ & & & & \\
\hline & $4(13 / 30)$ & $4(11 / 30)$ & $4(7 / 30)$ & $4(5 / 30)$ & $4(11 / 30)$ & $4(13 / 30)$ & $4(15 / 30)$ & $4(6 / 30)$ & $4(6 / 30)$ & $4(11 / 30)$ \\
\hline & $5(16 / 30)$ & $5(19 / 30)$ & $5(23 / 30)$ & $5(25 / 30)$ & $5(19 / 30)$ & $5(15 / 30)$ & $5(15 / 30)$ & $5(24 / 30)$ & $5(24 / 30)$ & $5(19 / 30)$ \\
\hline \multirow{3}{*}{80 kVp-FBP } & $3(8 / 30)$ & $3(2 / 30)$ & & & $3(2 / 30)$ & $3(9 / 30)$ & $3(2 / 30)$ & & & $3(3 / 30)$ \\
\hline & $4(21 / 30)$ & $4(26 / 30)$ & $4(13 / 30)$ & $4(12 / 30)$ & $4(22 / 30)$ & $4(21 / 30)$ & $4(26 / 30)$ & $4(15 / 30)$ & $4(16 / 30)$ & $4(22 / 30)$ \\
\hline & $5(1 / 30)$ & $5(2 / 30)$ & $5(17 / 30)$ & $5(18 / 30)$ & $5(6 / 30)$ & & $5(2 / 30)$ & $5(15 / 30)$ & $5(14 / 30)$ & $5(5 / 30)$ \\
\hline \multirow{3}{*}{80 kVp-S1 } & $3(7 / 30)$ & $3(2 / 30)$ & & & $3(2 / 30)$ & $3(6 / 30)$ & $3(1 / 30)$ & & & $3(2 / 30)$ \\
\hline & $4(17 / 30)$ & $4(17 / 30)$ & $4(12 / 30)$ & $4(13 / 30)$ & $4(20 / 30)$ & $4(16 / 30)$ & $4(19 / 30)$ & $4(13 / 30)$ & $4(12 / 30)$ & $4(19 / 30)$ \\
\hline & $5(6 / 30)$ & $5(11 / 30)$ & $5(18 / 30)$ & $5(17 / 30)$ & $5(8 / 30)$ & $5(8 / 30)$ & $5(10 / 30)$ & $5(17 / 30)$ & $5(18 / 30)$ & $5(9 / 30)$ \\
\hline \multirow{3}{*}{$80 \mathrm{kVp}-\mathrm{S2}$} & $3(5 / 30)$ & & & & $3(2 / 30)$ & $3(4 / 30)$ & & & & $3(1 / 30)$ \\
\hline & $4(19 / 30)$ & $4(19 / 30)$ & $4(13 / 30)$ & $4(12 / 30)$ & $4(20 / 30)$ & $4(18 / 30)$ & $4(19 / 30)$ & $4(12 / 30)$ & $4(14 / 30)$ & $4(21 / 30)$ \\
\hline & $5(6 / 30)$ & $5(11 / 30)$ & $5(17 / 30)$ & $5(18 / 30)$ & $5(8 / 30)$ & $5(8 / 30)$ & $5(11 / 30)$ & $5(18 / 30)$ & $5(16 / 30)$ & $5(8 / 30)$ \\
\hline \multirow{3}{*}{$80 \mathrm{kVp}-\mathrm{S3}$} & $3(3 / 30)$ & & & & & $3(3 / 30)$ & & & & \\
\hline & $4(20 / 30)$ & $4(18 / 30)$ & $4(12 / 30)$ & $4(12 / 30)$ & $4(19 / 30)$ & $4(19 / 30)$ & $4(19 / 30)$ & $4(13 / 30)$ & $4(13 / 30)$ & $4(20 / 30)$ \\
\hline & $5(7 / 30)$ & $5(12 / 30)$ & $5(18 / 30)$ & $5(18 / 30)$ & $5(11 / 30)$ & $5(8 / 30)$ & $5(11 / 30)$ & $5(17 / 30)$ & $5(17 / 30)$ & $5(10 / 30)$ \\
\hline \multirow{3}{*}{80 kVp-S4 } & $3(2 / 30)$ & & & & & $3(2 / 30)$ & & & & $3(1 / 30)$ \\
\hline & $4(18 / 30)$ & $4(15 / 30)$ & $4(12 / 30)$ & $4(14 / 30)$ & $4(19 / 30)$ & $4(19 / 30)$ & $4(16 / 30)$ & $4(13 / 30)$ & $4(13 / 30)$ & $4(19 / 30)$ \\
\hline & $5(10 / 30)$ & $5(15 / 30)$ & $5(18 / 30)$ & $5(16 / 30)$ & $5(11 / 30)$ & $5(9 / 30)$ & $5(14 / 30)$ & $5(17 / 30)$ & $5(17 / 30)$ & $5(10 / 30)$ \\
\hline \multirow{3}{*}{80 kVp-S5 } & $3(2 / 30)$ & & & & & $3(2 / 30)$ & & & & \\
\hline & $4(22 / 30)$ & $4(11 / 30)$ & $4(12 / 30)$ & $4(12 / 30)$ & $4(19 / 30)$ & $4(22 / 30)$ & $4(12 / 30)$ & $4(13 / 30)$ & $4(13 / 30)$ & $4(20 / 30)$ \\
\hline & $5(6 / 30)$ & $5(19 / 30)$ & $5(18 / 30)$ & $5(18 / 30)$ & $5(11 / 30)$ & $5(6 / 30)$ & $5(18 / 30)$ & $5(17 / 30)$ & $5(17 / 30)$ & $5(10 / 30)$ \\
\hline
\end{tabular}

Abbreviations: FBP, Filtered back projection; MIP, Maximum intensity projection; MPR, Multiplanar reformation images; S1-S5, SAFIRE strength level 1-5.

\begin{tabular}{|c|c|c|c|c|c|c|c|}
\hline \multirow[t]{2}{*}{ Review Item } & \multicolumn{7}{|c|}{ Kappa value (95\% Confidence Interval) } \\
\hline & $80 \mathrm{kVp}$-FBP & $80 \mathrm{kVp}-\mathrm{S1}$ & 80 kVp-S2 & $80 \mathrm{kVp}-\mathrm{S3}$ & $80 \mathrm{kVp}-\mathrm{S} 4$ & 80 kVp-S5 & 120 kVp-FBP \\
\hline overall image quality & $\begin{array}{c}0.848 \\
(0.655-1.000)\end{array}$ & $\begin{array}{c}0.857 \\
(0.702-1.000)\end{array}$ & $\begin{array}{c}0.729 \\
(0.511-0.948)\end{array}$ & $\begin{array}{c}0.918 \\
(0.761-1.000)\end{array}$ & $\begin{array}{c}0.940 \\
(0.824-1.000)\end{array}$ & $\begin{array}{c}0.933 \\
(0.804-1.000)\end{array}$ & $\begin{array}{c}0.888 \\
(0.744-0.917)\end{array}$ \\
\hline $\begin{array}{l}\text { Segmental displayed } \\
\text { in MIP }\end{array}$ & $\begin{array}{c}0.867 \\
(0.690-1.000)\end{array}$ & $\begin{array}{c}0.932 \\
(0.800-1.000)\end{array}$ & $\begin{array}{c}0.932 \\
(0.800-1.000)\end{array}$ & $\begin{array}{c}0.932 \\
(0.800-1.000)\end{array}$ & $\begin{array}{c}0.932 \\
(0.800-1.000)\end{array}$ & $\begin{array}{c}0.932 \\
(0.800-1.000)\end{array}$ & $\begin{array}{c}0.902 \\
(0.714-1.000)\end{array}$ \\
\hline $\begin{array}{l}\text { Segmental displayed } \\
\text { in MPR }\end{array}$ & $\begin{array}{c}0.737 \\
(0.505-0.968)\end{array}$ & $\begin{array}{c}0.795 \\
(0.575-1.000)\end{array}$ & $\begin{array}{c}0.865 \\
(0.686-1.000)\end{array}$ & $\begin{array}{c}0.932 \\
(0.800-1.000)\end{array}$ & $\begin{array}{c}0.933 \\
(0.803-1.000)\end{array}$ & $\begin{array}{c}0.932 \\
(0.800-1.000)\end{array}$ & $\begin{array}{c}0.889 \\
(0.676-1.000)\end{array}$ \\
\hline Diagnostic confidence & $\begin{array}{c}0.853 \\
(0.656-1.000)\end{array}$ & $\begin{array}{c}0.937 \\
(0.815-1.000)\end{array}$ & $\begin{array}{c}0.932 \\
(0.803-1.000)\end{array}$ & $\begin{array}{c}0.918 \\
(0.761-1.000)\end{array}$ & $\begin{array}{c}0.927 \\
(0.786-1.000)\end{array}$ & $\begin{array}{c}0.850 \\
(0.645-1.000)\end{array}$ & $\begin{array}{c}0.856 \\
(0.664-1.000)\end{array}$ \\
\hline
\end{tabular}

Abbreviations: FBP, Filtered back projection; MIP, Maximum intensity projection; MPR, Multiplanar reformation images; S1 S5, SAFIRE strength level 1 5.

tube voltage of $80 \mathrm{kVp}$ could reduce the radiation dose while providing satisfactory quality of image that could facilitate clinical diagnosis of renal artery CTA, which could optimize the scan and reconstruction parameters of CT protocol in the patients with renal mass whose BMI lower than $28 \mathrm{~kg} / \mathrm{m}^{2}$. 


\section{Acknowledgments}

We are thankful to Dr. Guanquan Shen and Dr. Xinqun Zeng who helped us to coordinate interpersonal relationships during this study.

\section{Footnotes}

Authors' Contributions: Pinggui Lei, data review, statistical evaluation, collection of references, writing, and correction of manuscript; Jun Jiao and Qinghong Duan, data collection, and manuscript correction; Xiaolin Wang, supervisor of this project; Hui Feng and Jujiang Mao, qualitative assessment for image quality; Xun Zou, data collection; Pingxian Wang, statistical evaluation and analysis.

Financial Disclosure: None declared.

Funding/Support: This study was supported by National Natural Science Foundation of China (NO. 81760312); Joint Fund of Guizhou Province Department of Science and Technology (NO. LH [2017]7208); Guizhou Province Clinical Discipline Subject Fund (NO. QWBF 2011[52]-03.

\section{References}

1. Israel GM, Silverman SG. The incidental renal mass. Radiol Clin North Am. 2011;49(2):369-83. doi: 10.1016/j.rcl.2010.10.007. [PubMed: 21333783].

2. Silverman SG, Israel GM, Herts BR, Richie JP. Management of the incidental renal mass. Radiology. 2008;249(1):16-31. doi: 10.1148/radiol.2491070783. [PubMed: 18796665].

3. Israel GM, Bosniak MA. How I do it: evaluating renal masses. Radiology. 2005;236(2):441-50. doi: 10.1148/radiol.2362040218. [PubMed: 16040900].

4. Mileto A, Nelson RC, Paulson EK, Marin D. Dual-Energy MDCT for Imaging the Renal Mass. AJR Am J Roentgenol. 2015;204(6):W640-7. doi: 10.2214/AJR.14.14094. [PubMed: 25730444].

5. Mileto A, Marin D, Nelson RC, Ascenti G, Boll DT. Dual energy MDCT assessment of renal lesions: an overview. Eur Radiol. 2014;24(2):35362. doi: 10.1007/s00330-013-3030-8. [PubMed: 24092045].

6. Brenner DJ, Hall EJ. Computed tomography-an increasing source of radiation exposure. $N$ Engl J Med. 2007;357(22):2277-84. doi: 10.1056/NEJMra072149. [PubMed: 18046031].

7. Schabel C, Fenchel M, Schmidt B, Flohr TG, Wuerslin C, Thomas C, et al. Clinical evaluation and potential radiation dose reduction of the novel sinogram-affirmed iterative reconstruction technique(SAFIRE) in abdominal computed tomography angiography. Acad Radiol. 2013;20(2):165-72. doi:10.1016/j.acra.2012.08.015. [PubMed: 23099242].

8. Baker ME, Dong F, Primak A, Obuchowski NA, Einstein D, Gandhi N, et al. Contrast-to-noise ratio and low-contrast object resolution on full- and low-dose MDCT: SAFIRE versus filtered back projection in a low-contrast object phantom and in the liver. AJR Am J Roentgenol. 2012;199(1):8-18. doi: 10.2214/AJR.11.7421. [PubMed: 22733888].

9. Li B, Behrman RH. Comment on the "report of AAPM TG 204: sizespecific dose estimates (SSDE) in pediatric and adult body CT examinations" [report of AAPM TG 204, 2011]. Med Phys. 2012;39(7):4613-4. author reply 4615-6. doi: 10.1118/1.4725756. [PubMed: 22830792].
10. Habib Geryes B, Calmon R, Khraiche D, Boddaert N, Bonnet D, Raimondi $F$. Radiation dose reduction in paediatric coronary computed tomography: assessment of effective dose and image quality. Eur Radiol. 2016;26(7):2030-8. doi: 10.1007/s00330-015-4032-5. [PubMed: 26433957].

11. Cohen MD. ALARA, image gently and CT-induced cancer. Pediatr Radiol. 2015;45(4):465-70. doi: 10.1007/s00247-014-3198-3. [PubMed: 25680877]

12. Shaw PV, Crouail P, Paynter R, Coeck M. Education and training in radiation protection: improving alara culture.J Radiol Prot. 2015;35(1):2237. doi: 10.1088/0952-4746/35/1/223. [PubMed: 25893232].

13. Oestreich AE. RSNA centennial article: ALARA 1912: "As low a dose as possible" a century ago. Radiographics. 2014;34(5):1457-60. doi 10.1148/rg.345130136. [PubMed: 25208291].

14. Schindera ST, Nauer C, Treier R, Trueb P, von Allmen G, Vock $\mathrm{P}$, et al. [Strategies for reducing the $\mathrm{CT}$ radiation dose]. Radiol ogy. 2010;50(12):1120. 1122-7. doi: 10.1007/s00117-010-2053-2. [PubMed: 20844858].

15. McCollough $\mathrm{CH}$, Primak AN, Braun N, Kofler J, Yu L, Christner J. Strategies for reducing radiation dose in CT. Radiol Clin North Am. 2009;47(1):27-40. doi: 10.1016/j.rcl.2008.10.006. [PubMed: 19195532].

16. Prakash P, Kalra MK, Digumarthy SR, Hsieh J, Pien H, Singh S, et al. Radiation dose reduction with chest computed tomography using adaptive statistical iterative reconstruction technique: initial experience. J Comput Assist Tomogr. 2010;34(1):40-5. doi 10.1097/RCT.0b013e3181b26c67. [PubMed: 20118720].

17. Mieville FA, Gudinchet F, Brunelle F, Bochud FO, Verdun FR. Iterative reconstruction methods in two different MDCT scanners: physical metrics and 4-alternative forced-choice detectability experiments-a phantom approach. Phys Med. 2013;29(1):99-110. doi: 10.1016/j.ejmp.2011.12.004. [PubMed: 22217444].

18. Kanematsu M, Kondo H, Miyoshi T, Goshima S, Noda Y, Tanahashi Y, et al. Whole-body CT with high heat-capacity X-ray tube and automated tube current modulation-effect of tube current limitation on contrast enhancement, image quality and radiation dose. Eur J Radiol. 2015;84(5):877-83. doi: 10.1016/j.ejrad.2015.01.019. [PubMed: 25740700].

19. Shen Y, Sun Z, Xu L, Li Y, Zhang N, Yan Z, et al. High-pitch, low-voltage and low-iodine-concentration CT angiography of aorta: assessment of image quality and radiation dose with iterative reconstruction. PLoS One. 2015;10(2). e0117469. doi: 10.1371/journal.pone.0117469. [PubMed: 25643353].

20. Chen GZ, Zhang LJ, Schoepf UJ, Wichmann JL, Milliken CM, Zhou $\mathrm{CS}$, et al. Radiation dose and image quality of $70 \mathrm{kVp}$ cerebral CT angiography with optimized sinogram-affirmed iterative reconstruction: comparison with $120 \mathrm{kVp}$ cerebral CT angiography. Eur Radiol. 2015;25(5):1453-63. doi: 10.1007/s00330-014-3533-y. [PubMed 25636415].

21. Solomon J, Samei E. Quantum noise properties of CT images with anatomical textured backgrounds across reconstruction algorithms: FBP and SAFIRE. Med Phys. 2014;41(9):91908. doi: 10.1118/1.4893497. [PubMed: 25186395].

22. Nie P, Li H, Duan Y, Wang X, Ji X, Cheng Z, et al. Impact of sinogram affirmed iterative reconstruction (SAFIRE) algorithm on image quality with $70 \mathrm{kVp}$-tube-voltage dual-source CT angiography in children with congenital heart disease. PLoS One. 2014;9(3). e91123. doi 10.1371/journal.pone.0091123. [PubMed: 24614683].

23. Silva AC, Lawder HJ, Hara A, Kujak J, Pavlicek W. Innovations in CT dose reduction strategy: application of the adaptive statistical iterative reconstruction algorithm. AJR Am J Roentgenol. 2010;194(1):191-9. doi: 10.2214/AJR.09.2953. [PubMed: 20028923]. 